\title{
The Diffuse Ionised Gas in NGC 4449
}

\author{
C. Muñoz-Tuñón ${ }^{1}$, O. Fuentes-Masip ${ }^{1}$ and H. O. Castañeda ${ }^{2}$ \\ ${ }^{1}$ Instituto de Astrofísica de Canarias, E-38200 La Laguna, Tenerife, Spain \\ cmt@iac.es; ofm@iac.es \\ ${ }^{2}$ ISO Science Operations Centre, PO Box 50727, E-28080 Madrid, Spain \\ hcastane@iso.vilspa.esa.es
}

Received 1997 August 1, accepted 1998 January 13

\begin{abstract}
It is now well established that there is a diffuse ionised medium which extends far beyond the disks of spirals. The mechanism responsible for the ionisation is still an issue of debate, and the method of measuring the total luminosity, independently of the observational set-up, has to be defined. Here we analyse the case of the Magellanic irregular NGC 4449, using Fabry-Perot bidimensional mapping of the $\mathrm{H} \alpha$ emission line, at the $4.2 \mathrm{~m}$ William Herschel Telescope (WHT) telescope on La Palma, Canary Islands. The total spatial coverage of $80 \times 80 \operatorname{arcsec}^{2}$ allows most of the star forming regions to be sampled at $0 \cdot 26$ arcsec/pixel spatial resolution. Using 3D spectroscopy, it is possible to define the individual star-forming regions and to produce a luminosity versus size diagram. This can be used to estimate directly the number of photons leaking out from those regions exceeding the thickness of the galactic disc. In the case of NGC 4449 it is shown that the large population of GEHRs can be the source of UV photons ionising the diffuse ionised gas (DIG) of the galaxy, and the $\log L-R$ diagram can be used to quantify the number of photons leaking out of the material surrounding the star forming regions. In the case of NGC 4449 it is found that $54 \%$ of the total luminosity of the giant star-forming regions is lost to the DIG. This implies a contribution of $3 \cdot 12 \times 10^{40} \mathrm{erg} \mathrm{s}^{-1}\left(1 \mathrm{erg} \mathrm{s}^{-1}=10^{-7}\right.$ $\mathrm{J} \mathrm{s}^{-1}$ ) which amounts to $30 \%$ of the galaxy total $\mathrm{H} \alpha$ luminosity.
\end{abstract}

Keywords: ISM: diffuse ionised gas — galaxies: individual (NGC 4449)

\section{Introduction}

We report part of a study of a sample of nearby Magellanic irregular galaxies with important star formation. The main interest in this class of objects is the large number of giant $\mathrm{H}$ II regions that each one contains. By studying each galaxy independently, the uncertainties in the galaxy distance determination are then ruled out and, therefore, the GEHRs in each galaxy constitute a homogeneous sample to test the luminosity versus velocity dispersion relationship, proposed as a distance indicator (Melnick 1979).

Observationally, the critical point is the precise determination of the parameters involved in the correlation; the determination of the size of the GEHRs, the measurement of emission line widths, and the luminosity, all require careful work, together with a discerning use of traditional methods. This has been an important fraction of the recent $\mathrm{PhD}$ work by Fuentes-Masip (1997) in which a complete catalogue of the $\mathrm{H}$ II regions of the galaxy NGC 4449 has been compiled. The case of Magellanic irregulars is particularly difficult because there are large numbers of star-forming entities which may overlap. The study, then, needs good spatial resolution to isolate individual regions, particularly if the bulk of the emission is engulfed by extended, low-intensity, ionised gas emission. This is the case for the galaxy NGC 4449 with an intense diffuse nebular luminosity present all over the optical body of the galaxy (Malumuth, Williams \& Schommer 1986; Scowen 1992) and 252 star-forming regions that have been catalogued (Sabbandin \& Bianchini 1979). Among them a large number have been classified as giant H II regions (Fuentes-Masip 1997). The optical angular size of the galaxy is $3.5 \times 2.5 \mathrm{arcmin}^{2}$, defined at the isocontour at $10^{-16} \mathrm{erg} \mathrm{cm}^{-2} \mathrm{~s}^{-1}$ for $\mathrm{H} \alpha$, which implies $5 \cdot 2 \times 3 \cdot 7 \mathrm{kpc}^{2}$, assuming a distance of $5 \mathrm{Mpc}\left(1^{\prime \prime}=25 \mathrm{pc}\right)$. It presents a moderately high star forming region (SFR) estimated as $1 \cdot 4-1.5 \mathrm{M}_{\odot} \mathrm{yr}^{-1}$. The extent of the $\mathrm{HI}$ is about 10 times the size of the stellar component and the total mass of gas is $6 \times 10^{9} \mathrm{M}_{\odot}$.

\section{Observations and Data Analysis}

We present results from the TAURUS-2 FabryPerot imaging spectrograph at the William Herschel Telescope $(4 \cdot 2 \mathrm{~m})$ at the ORM on La Palma (Canary Islands). The output from this set-up, 
after phase correction and calibration (Lewis \& Unger 1991), is a 3-D data cube with $x$ and $y$ being the spatial directions and $z$ the wavelength (etalon step) sampling. Data-cube dimensions were $(245,217,100)$ and the chosen etalon-FSR (free spectral range) was $13.4 \AA$ or $610 \mathrm{~km} \mathrm{~s}^{-1}$ in $\mathrm{H} \alpha$ $(\lambda=6562 \cdot 8 \AA)$. The spatial scale was $0 \cdot 26 \operatorname{arcsec}$ pixel $^{-1}$, or $6 \cdot 25 \mathrm{pc}$ at the distance on NGC 4449. The exposure time for each data-cube was one hour. Spectral scanning was $\Delta \lambda /$ step $=0 \cdot 2 \AA$ equivalent to $9 \mathrm{~km} \mathrm{~s}^{-1}$ in $\mathrm{H} \alpha$.

An image photon counting system (IPCS) was employed. With the IPCS the 100 planes are scanned continously with short exposures to avoid the effects of seeing variations during the total integration time. In this way, changes in seeing occurring during the 3600s exposure time, which would modify the emission-line profile, are minimised. During the observations seeing was about $1 \cdot 0^{\prime \prime}$. A complete description of the observations and data reduction is given in Fuentes-Masip (1997). Each TAURUS data-cube contains a total of 53165 spectra from an area of $1 \mathrm{arcmin}^{2}$, and hence the analysis procedure is an enormous task. Specific software, MATADOR, has been used for data analysis (see details in Gavryusev \& Muñoz-Tuñón 1996 and Muñoz-Tuñón, Garryuser \& Castañeda 1995).

Two overlapping fields in $\mathrm{H} \alpha$ were taken to cover an effective area of $80^{\prime \prime} \times 80^{\prime \prime}$. Interferential filter widths with FWHM $=15 \AA$ were used to limit the wavelength range, thus avoiding the overlapping of different interference orders.

The underlying continuum is fitted and subtracted from the calibrated data-cubes. The collapsed $\mathrm{H} \alpha$ map that results from adding all $z$ planes, free from continuum, has been used to unveil the ionised structure of the galaxy. Several independent methods were then used to isolate and catalogue individual $\mathrm{H}$ II regions, as well as to obtain their size and associated luminosity. The regions were identified in several ways: visually, detecting relative maxima of emission, using the flux gradient map and using the line-width and velocity gradient maps. In all cases the distribution function of the findings was computed to determine the possible errors and/or bias of each technique (see Fuentes-Masip 1997 for details). Flux calibration was carried out using an OIII CCD image taken at the $1 \mathrm{~m}$ Jacobus Kapteyn Telescope, also at the ORM. The complete catalogue will be published elsewhere (Fuentes-Masip et al. 1997a, b).

\section{Results and Discussion}

In Figure 1 we plot $\log \left(L_{\mathrm{H} \alpha}\right)$ versus radius, with the associated errors, for all the regions catalogued and measured in NGC 4449. For a classical H II region (i.e. in the absence of extinction and gas inhomogeneities) the $\log$ of the $\mathrm{H} \alpha$ luminosity is expected to be proportional to the log of the radius according to the relationship

$$
L \propto \frac{4}{3} \pi R^{3} \epsilon n^{2} \beta .
$$

We have performed a linear fit to the $\log (L)$ and $\log \left(R^{3}\right)$ values of the lower luminosity data points $\log (L)<38 \cdot 6$. The best fit to the physical model of classical Hir regions is given by

$$
\log (L)=38 \cdot 03+\log \left(R^{3}\right) .
$$

From Figure 1 two ranges, both in $R$ and in luminosity, can be distinguished. For radii larger than $R \approx 2$ arcsec or $\log L$ larger than $\approx 10^{38 \cdot 5} \mathrm{erg}$ $\mathrm{s}^{-1}$ (the giant $\mathrm{H}$ II regions, hereafter GHIIRs) most

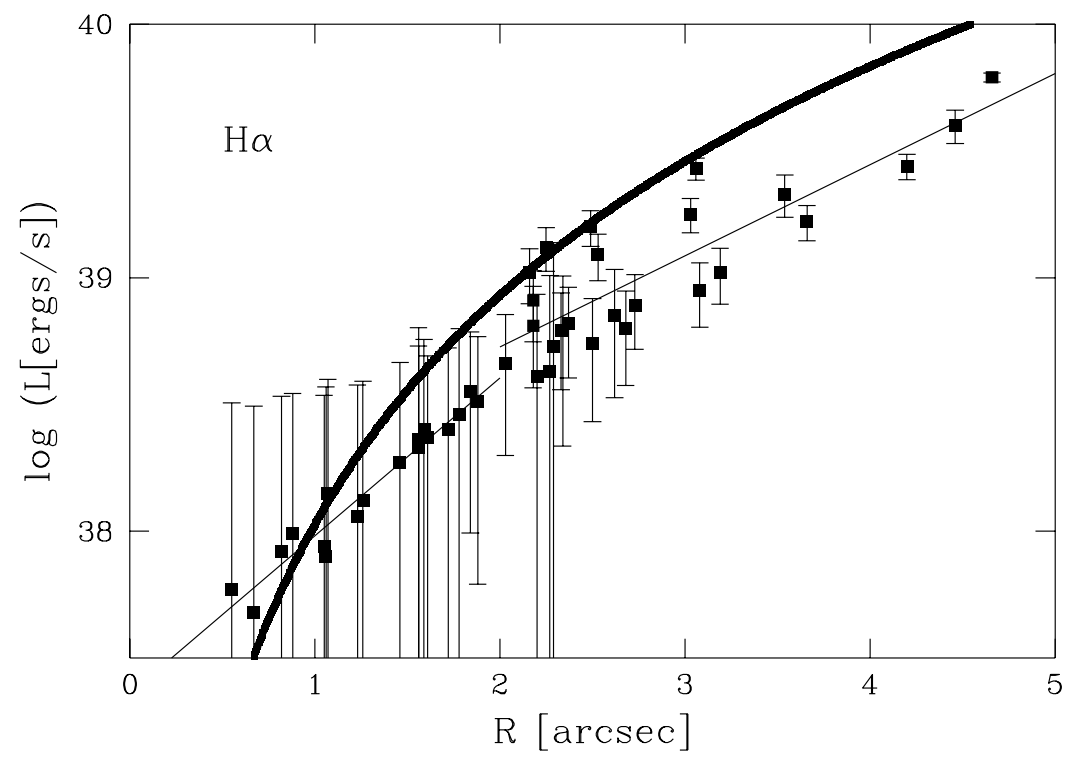

Figure 1-Overplotted on the observed data points is the function $\log (L)=38 \cdot 03+\log \left(R^{3}\right)$. 
$\mathrm{H}$ II regions are less luminous than expected. For the less luminous and smaller regions the increase of luminosity with size follows, within the errors, the trend expected for ionisation bound classical Strömgren spheres. This segregation is not related to any particular spatial distribution of the regions. Both classes, the smaller ones and the GHIIRs, are uniformly distributed in the galaxy. This also allows us to rule out any possible differential extinction as the cause of the leakage of photons coming from regions located in especially dusty areas. Also the possibility that the most luminous regions are also more heavily shrouded in dust is unlikely in dwarf irregulars known to be relatively metal-poor systems (see Kunth 1995, and references therein).

The change for the more luminous regions might be due to the fact that these regions have exceeded the thickness of the galactic disk and are therefore losing photons. These photons, leaking out from the large $\mathrm{H}$ II regions of the galaxy, might be an important source in ionising the DIG. The total luminosity, $L_{\text {total }}$, of the subsample is $\approx 2 \cdot 66 \times 10^{40}$ $\operatorname{erg~s}^{-1}$. If, on the other hand, one assumes that they should follow the same behaviour as the smaller $\mathrm{H}$ II regions, provided they are not losing photons, their luminosity, using equation ( 2 ), would be $\approx 5 \cdot 78 \times 10^{40}$ erg $\mathrm{s}^{-1}$. The difference is $3 \cdot 12 \times 10^{40} \mathrm{erg} \mathrm{s}^{-1}$ or $54 \%$ of the luminosity escaping from the GHIIRs and contributing to the DIG. The contribution to the total $\mathrm{H} \alpha$ luminosity coming from the other $\mathrm{H}$ II regions, those less luminous than $10^{38 \cdot 6} \mathrm{erg} \mathrm{s}^{-1}$, is $7 \cdot 03 \times 10^{39} \mathrm{erg} \mathrm{s}^{-1}$. Adding all these contributions, a total luminosity in $\mathrm{H} \alpha$ of $6.5 \times 10^{40} \mathrm{erg} \mathrm{s}^{-1}$ is obtained. This is lower that the $10^{41} \mathrm{erg} \mathrm{s}^{-1}$ reported by Hunter \& Gallagher (1986). The discrepancy comes from the fact that our observations cover only the central $80 \times 80 \operatorname{arcsec}^{2}$, whereas the galaxy extends more than twice as far. However, most GHIIRs of NGC 4449 are included in our data, and so if photons leaking from giant star formation complexes are the only source of the DIG luminosity the relative contribution of the DIG $\left(3 \cdot 12 \times 10^{40}\right.$ erg s$\left.{ }^{-1}\right)$ to the total measured $\mathrm{H} \alpha$ luminosity $\left(10^{41}\right.$ erg $\mathrm{s}^{-1}$ ) would be about $30 \%$.

Previous studies, using CCD images, to determine the DIG luminosity of NGC 4449 (Kennicutt, Edgar \& Hodge 1989; Hunter \& Gallagher 1990) from the total $\mathrm{H} \alpha$ luminosity of the galaxy give values of the relative contribution in the DIG of $50 \%$ and $20 \%$, respectively. The difference is probably due to the different lower isophotal surface-brightness cut used to define the DIG; the method is therefore also dependent on the exposure time and on the observational set-up.
Among the $44 \mathrm{H}$ II regions isolated and classified in this galaxy, the biggest, or brightest, larger than about 2 arcsec and with $L$ brighter than about $10^{38 \cdot 6} \mathrm{erg} \mathrm{s}^{-1}$, follow a linear relationship between $\log L$ and $\log R^{3}$ with a slope smaller than that for less luminous regions, departing from the expected behaviour of ionisation bound classical HII regions. This is considered as direct evidence of photons being lost from GEHRs, perhaps limited by the disk size and undergoing champagne flows. The boundaries of GHIIRs are ionised and champagne flows must be taking place. These can be an important source in providing the material detected as DIG in the galaxy NGC 4449. The evaporation of the GEHRs may well be the mechanism to supply the diffuse medium which bathes other irregulars with important star formation activity.

\section{Acknowledgments}

We express our thanks to Guillermo Tenorio-Tagle for his comments and fruitful discussions. The WHT is operated on the island of La Palma by the ING at the Observatorio del Roque de los Muchachos of the IAC. This study was partly financed by the DGICYT (grant PB94-1106), and the EEC through the Antares grant. We thank the workshop organisers, in particular Quentin Parker, for their kindness and hospitality. We would also like to acknowledge our anonymous referee for contributing towards improving the content of the paper.

Fuentes-Masip, O. 1997, PhD thesis, Univ. La Laguna

Fuentes-Masip, O., et al. 1997a, ApJ, submitted

Fuentes-Masip, O., et al. 1997b, ApJ, submitted

Gavryusev, V., \& Muñoz-Tuñón, C. 1996, Astronomical Data Analysis Software and Systems V. ASP, 101

Hunter, D. A., \& Gallagher, J. S. 1986, PASP, 98, 5

Hunter, D. A., \& Gallagher, J. S. 1990, ApJ, 362, 480

Hunter, D. A., \& Gallagher, J. S. 1996, ApJ,

Kennicutt, R. C., Edgar, B. K., \& Hodge, P. W. 1989, ApJ, 337,761

Kunth, D. 1995, in The Interplay between Massive Star Formation, the ISM and Galaxy Evolution, ed. D. Kunth et al. (Paris: Editions Frontieres)

Lewis, J., \& Unger, S. 1991, William Herschel Telescope TAURUS data and how to reduce it

Malumuth, E. M., Williams, T. B., \& Schommer, R. A. 1986, AJ, 91, 1295

Melnick, J. 1979, ApJ, 228, 112

Muñoz-Tuñón, C., Gavryusev, V., \& Castañeda, H. O. 1995, AJ, 110, 1630

Sabbandin, F., \& Bianchini, A. 1979, PASP, 91, 280

Scowen, P. A. 1992, PhD thesis, Rice University, Houston 